\section{Surface Superconductivity in Niobium for Superconducting RF Cavities}

\section{S. Casalbuoni ${ }^{1,2}$, E.-A. Knabbe ${ }^{2}$, J. Kötzler ${ }^{1}$, L. Lilje ${ }^{2}$,} L. von Sawilski ${ }^{1}$, P. Schmüser ${ }^{3}$, B. Steffen ${ }^{2,3}$

${ }^{1}$ Institut für Angewandte Physik, Universität Hamburg

${ }^{2}$ Deutsches Elektronensynchrotron DESY, Hamburg

${ }^{3}$ Institut für Experimentalphysik, Universität Hamburg

\section{Abstract}

A systematic study is presented on the superconductivity (sc) parameters of the ultrapure niobium used for the fabrication of the nine-cell $1.3 \mathrm{GHz}$ cavities for the linear collider project TESLA. Cylindrical Nb samples have been subjected to the same surface treatments that are applied to the TESLA cavities: buffered chemical polishing (BCP), electrolytic polishing (EP), low-temperature bakeout (LTB). The magnetization curves and the complex magnetic susceptibility have been measured over a wide range of temperatures and dc magnetic fields, and also for different frequencies of the applied ac magnetic field. The bulk superconductivity parameters such as the critical temperature $T_{c}=9.26 \mathrm{~K}$ and the upper critical field $B_{c 2}(0)=410 \mathrm{mT}$ are found to be in good agreement with previous data. Evidence for surface superconductivity at fields above $B_{c 2}$ is found in all samples. The critical surface field exceeds the Ginzburg-Landau field $B_{c 3}=1.695 B_{c 2}$ by about $10 \%$ in BCP-treated samples and increases even further if EP or LTB are applied. From the field dependence of the susceptibility and a power-law analysis of the complex ac conductivity and resistivity the existence of two different phases of surface superconductivity can be established which resemble the Meissner and Abrikosov phases in the bulk: (1) "coherent surface superconductivity", allowing sc shielding currents flowing around the entire cylindrical sample, for external fields $B$ in the range $B_{c 2}<B<B_{c 3}^{c o h}$, and (2) "incoherent surface superconductivity" with disconnected sc domains for $B_{c 3}^{c o h}<B<B_{c 3}$. The "coherent" critical surface field separating the two phases is found to be $B_{c 3}^{c o h}=0.81 B_{c 3}$ for all samples. The exponents in the power law analysis are different for $\mathrm{BCP}$ and $\mathrm{EP}$ samples, pointing to different surface topologies.

\section{Introduction}

The proposed linear electron-positron collider project TESLA is based on superconductor technology for particle acceleration. For a centre-of-mass energy of $500 \mathrm{GeV}$ an accelerating field of $23.4 \mathrm{MV} / \mathrm{m}$ is required in the $1.3 \mathrm{GHz}$ nine-cell cavities which are made from pure niobium and cooled by superfluid helium at $2 \mathrm{~K}$. The cavities for the TESLA Test Facility (TTF) linac are fabricated from $2.8 \mathrm{~mm}$ thick niobium sheets by deep drawing and electron beam welding. A damage layer of about $150 \mu \mathrm{m}$ thickness is removed from the inner surface to obtain optimum performance in the superconducting state. For the TTF cavities this has been done so far by chemical etching which consists of two alternating processes: dissolution of the natural $\mathrm{Nb}_{2} \mathrm{O}_{5}$ layer by $\mathrm{HF}$ and re-oxidation of the niobium by a strongly oxidizing acid such as nitric acid $\left(\mathrm{HNO}_{3}\right)[1,2]$. To reduce the etching speed a buffer substance is added, for example phosphoric acid, and the mixture is cooled below $15^{\circ} \mathrm{C}$. The standard procedure with a removal rate of about $1 \mu \mathrm{m}$ per minute is called buffered chemical polishing (BCP) using an acid mixture of $\mathrm{HF}(40 \%), \mathrm{HNO}_{3}$ $(65 \%)$ and $\mathrm{H}_{3} \mathrm{PO}_{4}(85 \%)$ in a volume ratio of $1: 1: 2$. In the most recent industrial production of 24 TTF cavities an average gradient $26.1 \pm 2.3 \mathrm{MV} / \mathrm{m}$ at a quality factor $Q_{0}=1 \cdot 10^{10}$ was achieved. The technology developed for TTF is hence adequate for TESLA-500 but considerable improvements are needed for an upgrade of the collider to $800 \mathrm{GeV}$. A detailed description of the present status of the nine-cell cavity layout, fabrication, preparation and tests can be found in [3].

After many years of intensive $R \& D$ there exists now compelling evidence that the BCP process limits the attainable field in multi-cell niobium cavities to about $30 \mathrm{MV} / \mathrm{m}$. This is significantly below the physical limit of about $45 \mathrm{MV} / \mathrm{m}$ which is given by the condition that the radio frequency (rf) magnetic field has to stay below the critical field of the superconductor. For the type II superconductor niobium the maximum tolerable rf field appears to be close to the thermodynamic critical field ( $B_{c} \approx 190 \mathrm{mT}$ at 2 Kelvin).

An alternative surface preparation method is electrolytic polishing (EP). The material is removed in an acid mixture (for example $\mathrm{HF}$ and $\mathrm{H}_{2} \mathrm{SO}_{4}$ ) under the flow of an electric current. Sharp edges or tips are smoothed out and a very glossy surface can be obtained. Using electrolytic polishing, scientists at the KEK laboratory in Tsukuba (Japan) achieved gradients of up to $40 \mathrm{MV} / \mathrm{m}$ in single-cell cavities $[4,5]$. Meanwhile gradients of $35-40$ $\mathrm{MV} / \mathrm{m}$ have been obtained repeatedly in many $1.3 \mathrm{GHz}$ single-cell test cavities [6-8]. Recently the EP technology has been successfully transferred to the nine-cell TESLA cavities yielding a record value of $39 \mathrm{MV} / \mathrm{m}$ in a multicell cavity [9].

The superiority of EP as compared to $\mathrm{BCP}$ can be partially understood in terms of the much reduced surface roughness. The sharp ridges at the grain boundaries of an etched niobium surface may cause local enhancements of the rf magnetic field and thereby lead to a premature breakdown of superconductivity at these localized spots. A model based on this idea, developed by Knobloch et al. [10], is able to explain the reduction of the quality factor at high field. However, a puzzling observation which does not fit into this geometrical picture was made during the CERN-DESY-Saclay $\mathrm{R} \& \mathrm{D}$ programme on the electropolishing of single-cell cavities [8]: after the EP and rinsing with ultrapure 
water the cavities failed to reach full performance but exhibited a strong decrease of quality factor when high fields were approached. Applying a 24 - 48 hour "bakeout" at $120-140^{\circ} \mathrm{C}$ to the evacuated cavity resulted in a dramatic improvement: very high gradients were accessible and the "Q drop" vanished. It should be noted that the EP treatment at KEK $[4,5]$ already included a bakeout at $85^{\circ} \mathrm{C}$. The BCS surface resistance at $1.3-1.5 \mathrm{GHz}$ is found to be reduced by a factor of 2 after baking $[8,11]$. However, by removing a layer of $\sim 200-300 \mathrm{~nm}$ in steps of $\sim 50 \mathrm{~nm}$ from the EP surface by "oxipolishing" the reduction in $R_{B C S}$ is lost and the "Q drop" reappears [11]. The reduction of $R_{B C S}$ during baking has been attributed to oxygen atoms diffusing either from the dielectric $\mathrm{Nb}_{2} \mathrm{O}_{5}$ layer or from intergrain niobium oxides/suboxides down to a depth of 200-300 nm [11]. For further discussions we refer to the review talks by P. Kneisel [12] and B. Visentin [13] at the SRF2003 Workshop. Relevant investigations on the influence of surface treatment on single cell cavities have been presented at the same workshop by Ciovati et al. [16].

Another explanation for the superiority of electropolished cavities has been proposed by Halbritter [17]. The smoother surface and the cleaner grain boundaries of an EP-treated cavity may lead to a reduction of dielectric surface losses which are caused by interface tunnel exchange between the conduction electrons in $\mathrm{Nb}$ and localized states in the $\mathrm{Nb}-\mathrm{Nb}_{2} \mathrm{O}_{5-x}$ interface.

Magnetic measurements on niobium samples are a useful tool to explore the surface treatments which improve cavity performance. This idea is based on the fact that for pure niobium the ratio $\kappa=\lambda_{L} / \xi$ is in the order of unity, so that surface superconductivity and electromagnetic losses of microwave fields reside in thin surface sheaths of nearly the same thickness, given by the correlation length $\xi$ and the penetration depth $\lambda_{L}$, respectively. The experimental studies on the magnetization and susceptibility of niobium samples presented here have been carried out with the aim to gain an understanding of the superconducting properties in this sheath and their dependence on the various treatments (BCP, EP, bakeout) that are applied to the TESLA cavities. The samples have been cut from remainders of the niobium sheets used in cavity production and have been subjected to the same chemical, electro-chemical and thermal treatments as the TESLA cavities.

\section{Experimental Procedure}

The samples for the magnetization and susceptibility measurements are cylinders with a raw diameter of $2.5 \mathrm{~mm}$ and a height of $2.8 \mathrm{~mm}$ which are cut by electroerosion from the $\mathrm{Nb}$ sheets used for $\mathrm{rf}$ cavity production. The niobium is of high purity with a residual resistiv- ity ratio $R R R$ of 300 . The impurity contents is given in Table 1 The electroerosion leaves an oxide surface layer of several $\mu \mathrm{m}$ thickness which is removed by chemical etching of about $50 \mu \mathrm{m}$, applying the standard BCP process. The samples are rinsed with ultrapure water and annealed for 2 hours at $800{ }^{\circ} \mathrm{C}$ in a vacuum furnace $\left(p<10^{-7}\right.$ mbar) to remove dissolved hydrogen and relieve mechanical stress in the material. After the annealing a second $50 \mu \mathrm{m} \mathrm{BCP}$ and water rinsing is applied. In this state the samples have a similar surface structure as the BCP-treated cavities.

Table 1: Impurity contents of the Nb samples.

\begin{tabular}{|c|c||c|c|}
\hline $\mathrm{Ta}$ & $210-950 \mathrm{ppm}$ & $\mathrm{O}$ & $<40 \mathrm{ppm}$ \\
\hline $\mathrm{W}$ & $<100 \mathrm{ppm}$ & $\mathrm{N}$ & $<20 \mathrm{ppm}$ \\
\hline $\mathrm{Mo}$ & $<50 \mathrm{ppm}$ & $\mathrm{C}$ & $<20 \mathrm{ppm}$ \\
\hline $\mathrm{Ti}$ & $<40 \mathrm{ppm}$ & $\mathrm{H}$ & $<3 \mathrm{ppm}$ \\
\hline $\mathrm{Fe}$ & $<30 \mathrm{ppm}$ & & \\
\hline
\end{tabular}

Several samples have been electropolished after the BCP treatment. Only the cylindrical surface has been polished with a removed thickness between $40 \mu \mathrm{m}$ and $165 \mu \mathrm{m}$. The EP has been carried out at room temperature in a mixture of $\mathrm{HF}(56 \%)$ and $\mathrm{H}_{2} \mathrm{SO}_{4}(90 \%)$. Low temperature baking (LTB) has been applied both to BCP- and EP-treated samples, with a bakeout temperature of $100 \pm 1^{\circ} \mathrm{C}, 120 \pm 5^{\circ} \mathrm{C}, 123 \pm 1^{\circ} \mathrm{C}$ resp. $144 \pm 1^{\circ} \mathrm{C}$ and a baking time between 12 and 96 hours in a vacuum furnace at $p<10^{-7}$ mbar. Three of the BCP-treated and baked samples have been subsequently etched by $1 \mu \mathrm{m}$, $5 \mu \mathrm{m}$ and $10 \mu \mathrm{m}$ in order to investigate whether possible baking effects are lost by the removal of the surface layer. Scanning Electron Microscopy and Atomic Force Microscopy [18] reveal a very low surface roughness of about $1 \mathrm{~nm}$ on the $\mathrm{Nb}$ grains (area of about $10 \times 10 \mu \mathrm{m}^{2}$ ). The steps at grain boundaries range from $1 \mu \mathrm{m}$ to a few $\mu \mathrm{m}$ on BCP-treated samples [19] and are below $100 \mathrm{~nm}$ after the EP.

The sample magnetization is determined with a commercial SQUID magnetometer (Quantum Design $\mathrm{MPMS}_{2}$ ) at temperatures ranging from $2 \mathrm{~K}$ to $300 \mathrm{~K}$ in external dc fields between zero and 1 Tesla. The SQUID magnetometer allows also to measure the ac susceptibility at $10 \mathrm{~Hz}$. A second magnetometer, based on the mutual inductance technique, is used in combination with a lock-in amplifier (EG\&G PARC Model 5302) to extend the ac susceptibility measurements up to frequencies of $1 \mathrm{MHz}$ in the temperature range between $1.5 \mathrm{~K}$ and $4.2 \mathrm{~K}$ (pumped ${ }^{4} \mathrm{He}$ cryostat). The linearity of the response to the applied ac field has been verified for ac field amplitudes from $0.1 \mu \mathrm{T}$ to $500 \mu \mathrm{T}$. In all measurements the external magnetic fields (dc and ac) are carefully aligned parallel to the symmetry axis of the cylinders, so that above $H_{c 2}$ surface superconductivity can only nucleate on the cylindrical walls and not on the end faces. The demagnetization factor $N_{Z}=0.36$ derived from the initial slope of 
the dc magnetization curve $M(H)=-H /\left(1-N_{Z}\right)$ agrees with the theoretical expression $N_{Z}=1-1 /(1+q a / b)[20]$. Here $q=4 / 3 \pi+2 / 3 \pi \tanh (1.27 b / a \ln (1+a / b))$, where $h=2 b$ is the height and $a$ the radius of the cylinder. The magnetization and susceptibility data presented in the next sections have been corrected using this demagnetization factor.

\section{Bulk superconductivity}

The superconducting transition temperature $T_{c}$ is determined from the onset of the screening component $\chi^{\prime}$ of the complex ac susceptibility $\chi=\chi^{\prime}-i \chi^{\prime \prime}$, measured at vanishing dc field as a function of temperature (see Fig. 1). The average critical temperature $T_{c}=9.263 \pm 0.003 \mathrm{~K}$ of all samples agrees with the value $T_{c}=9.25 \pm 0.01 \mathrm{~K}$ reported by Finnemore at al. [21] for high purity $\mathrm{Nb}(R R R=1600 \pm 400)$.

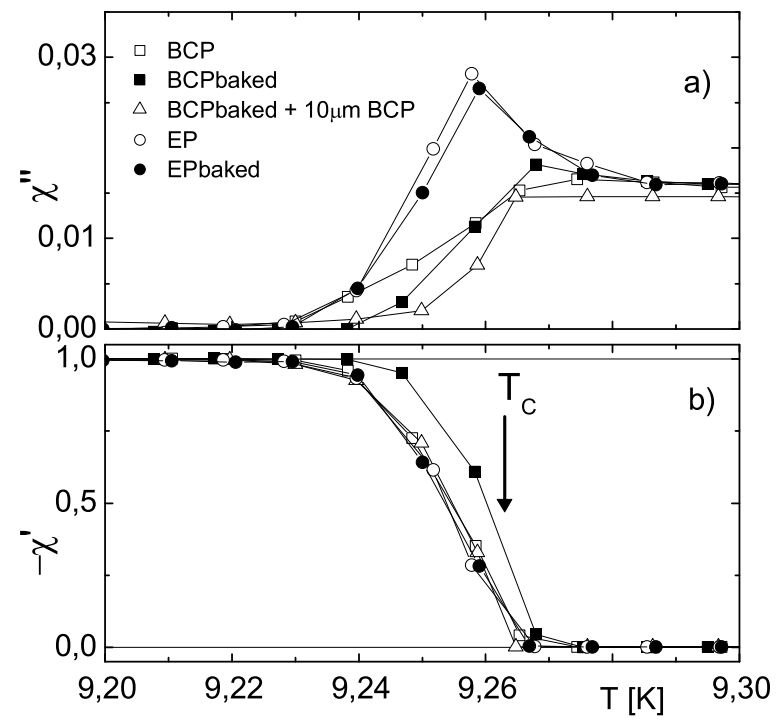

Figure 1: (a) Imaginary and (b) real part of the linear ac susceptibility recorded near the zero-field transition temperature of the Nb-cylinders under investigation. Note the different $\chi^{\prime \prime}$-scale in (a).

The complex ac susceptibility ${ }^{1} \chi(\omega)=\chi^{\prime}(\omega)-i \chi^{\prime \prime}(\omega)$ is related to the conductivity of a cylinder of radius $a$ by $[22,23]$

$$
\begin{aligned}
\chi^{\prime}(\omega)-i \chi^{\prime \prime}(\omega) & =\frac{2 I_{1}(x)}{x I_{0}(x)}-1 \\
\text { with } x & =\sqrt{i \omega a^{2} \mu_{0}\left(\sigma^{\prime}(\omega)-i \sigma^{\prime \prime}(\omega)\right)} .
\end{aligned}
$$

Here $I_{0}(x)$ and $I_{1}(x)$ are modified Bessel functions. For a short cylinder with heigth $h=2 a$ the radius must be replaced by $a / \sqrt{2}^{2}$. The complex conductivity $\sigma(\omega)$ is obtained from the measured susceptibility $\chi(\omega)$ by means of

\footnotetext{
${ }^{1}$ The sign of the imaginary part refers to a time dependence $\exp (+i \omega t)$ of the electromagnetic fields.

2 The effect of sample geometry on the relation between $\chi(\omega)$ and $\sigma(\omega)$ is extensively studied in Ref. [25].
}

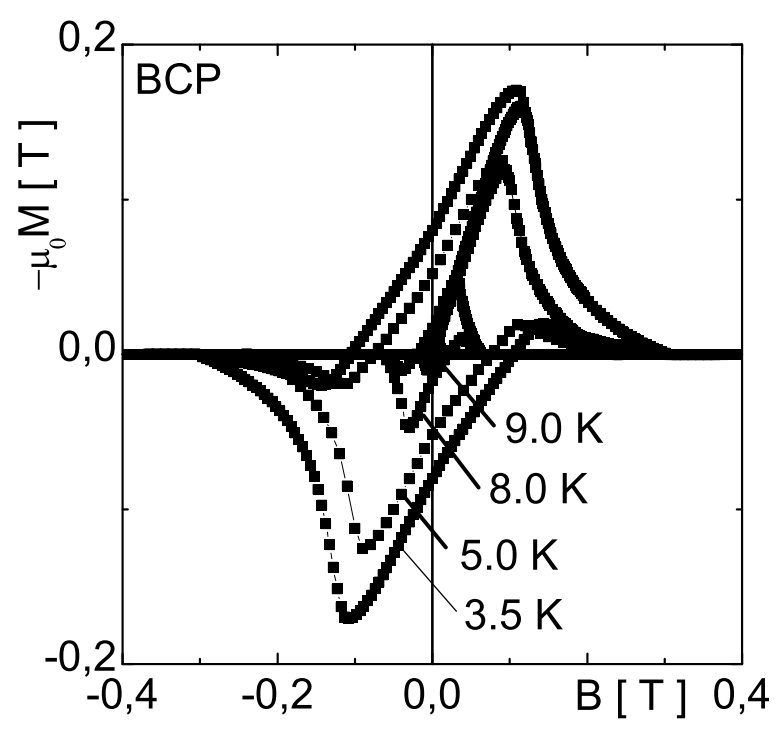

Figure 2: Magnetization isothermal loops for a BCP sample. Plotted is $-\mu_{0} M$ as function of the applied field $B=\mu_{0} H$.

an inversion routine [24]. The average Ohmic resistivity at $10 \mathrm{~Hz}$ and $T>T_{c}$ is found to be $\rho_{n}=(0.5 \pm 0.1) \mathrm{n} \Omega \mathrm{m}$, confirming the purity of the samples and the specification of the manufacturers of $R R R \simeq 300$.

Table 2: Parameters of the Nb samples.

\begin{tabular}{|l|c|c|}
\hline & BCP & EP \\
\hline$T_{c}[\mathrm{~K}]$ & \multicolumn{2}{|c|}{$9.263 \pm 0.003$} \\
\hline$R R R$ & \multicolumn{2}{|c|}{$\approx 300$} \\
\hline $\begin{array}{l}\text { surf. roughness } \\
\text { on grain [nm] }\end{array}$ & \multicolumn{2}{|c|}{$\approx 1$} \\
\hline steps at grain bound. & $1-5 \mu \mathrm{m}$ & $\lesssim 0.1 \mu \mathrm{m}$ \\
\hline$B_{c}(0)[\mathrm{mT}]$ & \multicolumn{2}{|c|}{$180 \pm 5$} \\
\hline$B_{c 2}(0)[\mathrm{mT}]$ & \multicolumn{2}{|c|}{$410 \pm 5$} \\
\hline$J_{c}(0,0)\left[\mathrm{A} / \mathrm{mm}^{2}\right]$ & $240 \pm 10$ & $180 \pm 10$ \\
\hline
\end{tabular}

In order to determine the thermodynamic critical field $H_{c}$ the reversible magnetization $M_{\text {rev }}=\left(M_{+}+M_{-}\right) / 2$ is deduced from the hysteresis loops (Fig. 22), where $M_{+}$ corresponds to the ascending and $M_{-}$to the descending branch of the loop. The reversible magnetisation of a BCP sample is plotted in Fig. 3a. The thermodynamic critical field is given by

$$
H_{c}^{2}=2 \mu_{0} \int_{0}^{H_{c 2}} M_{r e v}(H) d H .
$$

The critical field at $T=0$ is derived using the empirical law

$$
H_{c}(T)=H_{c}(0)\left(1-\left(T / T_{c}\right)^{2}\right)
$$

where $T_{c}$ is given in Tab. 22 The upper critical field $H_{c 2}$ can be determined with high accuracy from the onset of the irreversible magnetization $M_{i r r}=\left(M_{+}-M_{-}\right) / 2$, as 

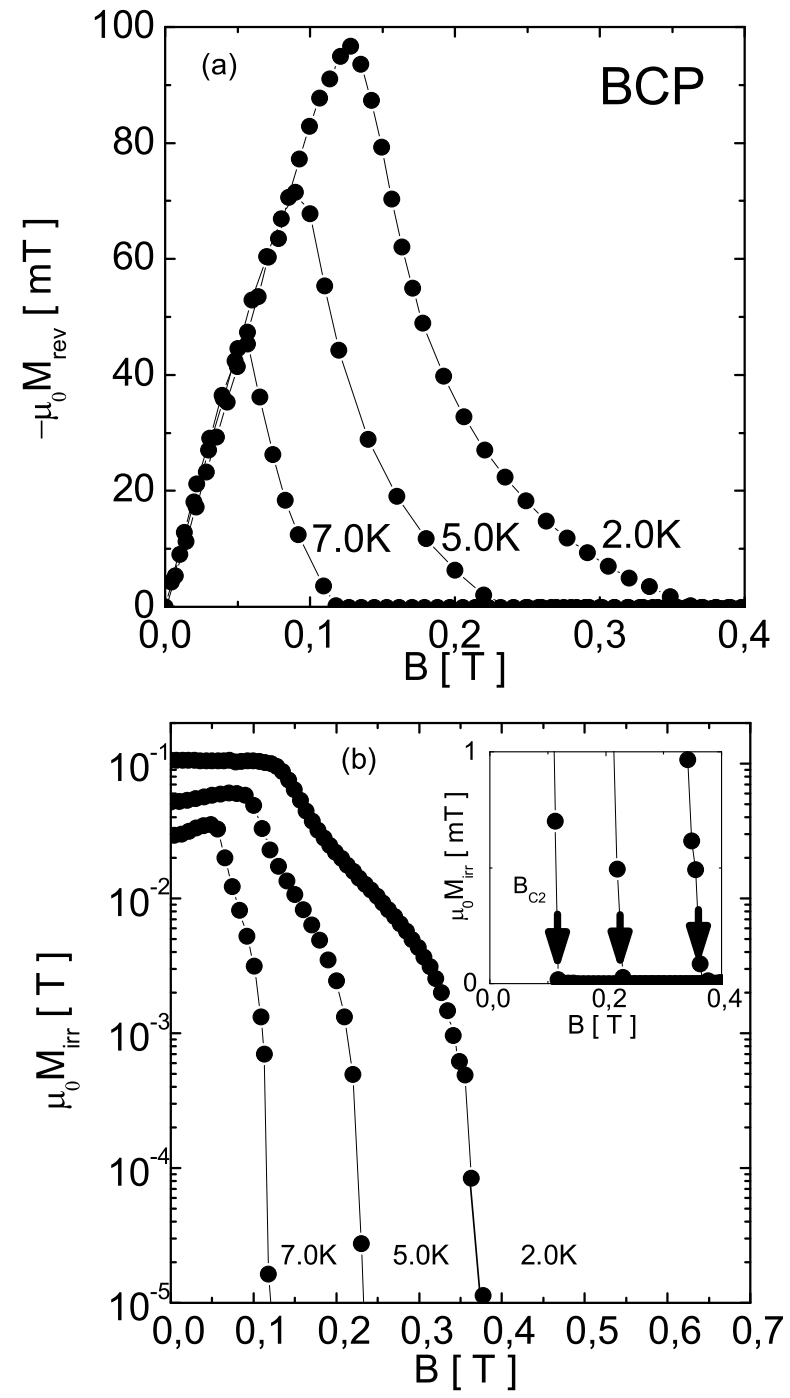

Figure 3: (a) Reversible magnetization of a BCP sample at $2-7 \mathrm{~K}$, evaluated from the isothermal loops. (b) Irreversible magnetization at 2, 5 and $7 \mathrm{~K}$, showing the sharp onset of bulk superconductivity at $B_{c 2}(T)=\mu_{0} H_{c 2}(T)$.

shown in the inset of Fig. 3 $\mathrm{b}$. The $H_{c 2}$ data are well described by the temperature dependence [26]

$$
H_{c 2}(T)=H_{c 2}(0) \frac{\left(1-\left(T / T_{c}\right)^{2}\right)}{\left(1+\left(T / T_{c}\right)^{2}\right)} .
$$

The thermodynamic and upper critical fields ${ }^{3}$ are plotted in Fig. 4 as functions of temperature. Averaged over all samples we get $B_{c 2}(0)=\mu_{0} H_{c 2}(0)=410 \pm 5 \mathrm{mT}$ and $B_{c}(0)=\mu_{0} H_{c}(0)=180 \pm 5 \mathrm{mT}$ (see also Tab. 2). While $B_{c 2}(0)$ agrees with the upper critical field reported in Ref. [21] for pure niobium, our $B_{c}(0)$ is $10 \%$ smaller than the value $B_{c}(0)=199 \pm 1 \mathrm{mT}$ quoted in [21].

Within the Ginzburg-Landau (GL) theory it is possible to determine the GL parameter at zero temperature $\kappa(0)=B_{c 2}(0) /\left(\sqrt{2} B_{c}(0)\right)=1.61 \pm 0.07$, the GL

\footnotetext{
${ }^{3}$ In this paper we use the SI unit system and quote magnetic fields in the form $B=\mu_{0} H$ with the unit Tesla.
}

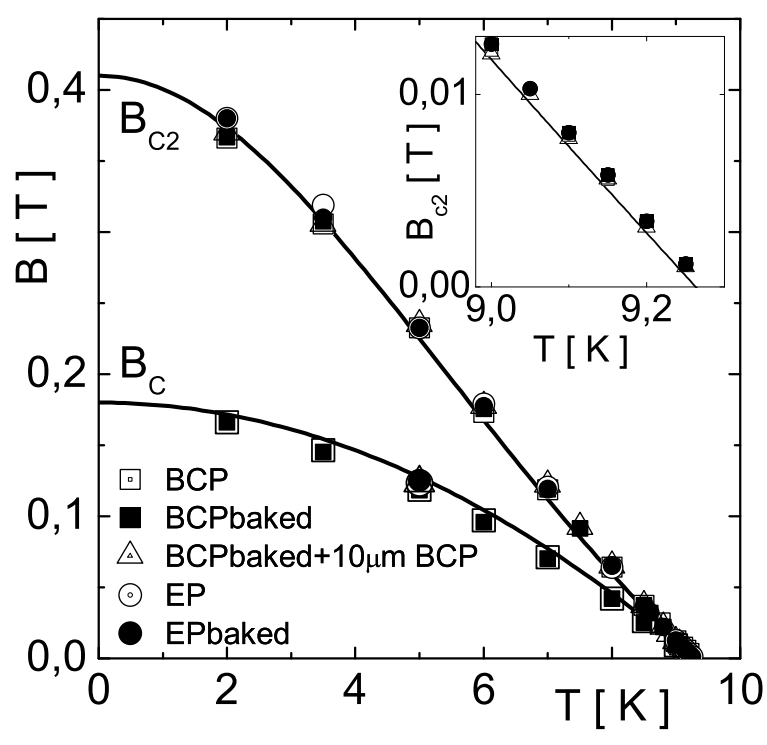

Figure 4: Temperature variation of the upper critical field $B_{c 2}=\mu_{0} H_{c 2}$ and of the thermodynamic critical field $B_{c}$ of all cylinders. Solid curves: fits based on Eqs. (3.3) and (3.4). The expected linear behaviour of $B_{c 2}$ close to $T_{c}$ is displayed in the inset.

coherence length $\xi(0)=\sqrt{\hbar /\left(2 e B_{c 2}(0)\right)}=28.3 \pm 0.2 \mathrm{~nm}$ and the London penetration depth $\lambda_{L}(0)=\kappa(0) \xi(0)=$ $46 \pm 2 \mathrm{~nm}$. The data from differently treated samples are in good agreement and confirm the expectation that neither electropolishing nor low-temperature bakeout change the superconductor parameters of the bulk niobium.

The hysteresis loops observed in the magnetization measurements are a clear proof of magnetic flux pinning. Under the assumption that pinning in the bulk dominates one can derive the critical current density from the irreversible part of the magnetization: $J_{c}=3 M_{i r r} / a$, where $a$ is the radius of the $\mathrm{Nb}$ cylinder. We obtain $J_{c}=240$ $\mathrm{A} / \mathrm{mm}^{2}$ for the BCP samples and $J_{c}=180 \mathrm{~A} / \mathrm{mm}^{2}$ for the EP samples (both at $T=4.2 \mathrm{~K}$ and $B=0$ ). Since $\mathrm{EP}$ does not affect the bulk this difference implies that surface flux pinning plays an important role too. Similar observations were made by De Sorbo [27] who found large variations of $J_{c}$ between 10 and $1000 \mathrm{~A} / \mathrm{mm}^{2}$ for $\mathrm{Nb}$ with $R R R \simeq 500$, depending on the mechanical and thermal treatment.

\section{Surface superconductivity}

\subsection{Critical surface field}

Close to the surface a type II superconductor remains in the superconducting state even if the upper critical field of the bulk is exceeded. According to the Ginzburg-Landau theory the critical surface field is given by $B_{c 3}=1.695 B_{c 2}[28]$. Owing to the high sensitivity of the SQUID magnetometer, evidence for surface super- 


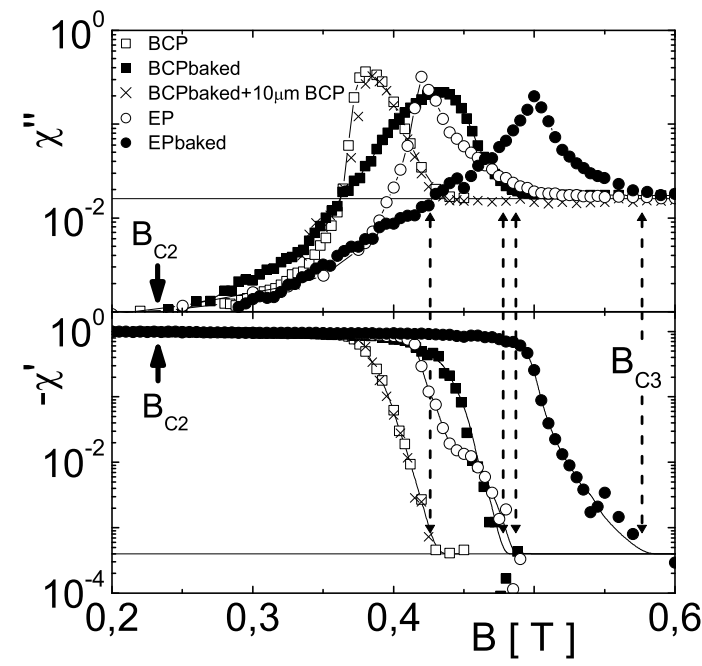

Figure 5: Field dependence of the ac susceptibility on a logarithmic scale of $\mathrm{Nb}$ cylinders with different surface conditions. The data have been taken at $T=5.0 \mathrm{~K}, f=10 \mathrm{~Hz}$ and $B_{a c}=1 \mu \mathrm{T}$. The surface nucleation fields $B_{c 3}$ (arrows) are defined by the onset of an excess absorption $\left(\chi^{\prime \prime}-\chi_{n}^{\prime \prime}\right)$ or excess shielding $\left(\chi^{\prime}-\chi_{n}^{\prime}\right)$, where $\chi_{n}^{\prime \prime}$ resp. $\chi_{n}^{\prime}$ are the imaginary resp. real part of $\chi$ in the normal state (indicated by the horizontal solid lines).

conductivity in the field range $B_{c 2}<B<B_{c 3}$ is in fact observable in all samples. In Fig. 5 we show the complex ac susceptibility $\chi^{\prime}-i \chi^{\prime \prime}$ at $5.0 \mathrm{~K}$ and $10 \mathrm{~Hz}$ as a function of the applied dc field for samples with different surface treatments: BCP, EP, baked and unbaked.

Approaching the transition to superconductivity from the normal state, i.e. from high fields, the nucleation field $B_{c 3}$ is commonly defined $[29,30]$ by the onset of screening, i.e., the appearance of a finite $\left|\chi^{\prime}\right|$ above the suceptibility value $\chi_{n}^{\prime}$ in the normal state. We observe a strong dependence of $B_{c 3}$ on the surface condition: the $\mathrm{BCP}$ sample has the lowest $B_{c 3}$, and both EP and baking increase the critical surface field.

The ac susceptibility of a BCP sample at temperatures between $3.5 \mathrm{~K}$ and $9.0 \mathrm{~K}$ is plotted in Fig. 6. Similar measurements have been performed for all samples. The temperature dependence of $B_{c 3}$ for samples with different surface treatment is shown in Fig. 7 The data are well described by the relation

$$
B_{c 3}(T)=r_{32} \cdot B_{c 2}(T)
$$

where the ratio $r_{32}$ depends on the surface preparation but not on the temperature, see Tab. 3 and Fig. 8

The reproducibility of the measurements of the ratio $r_{32}$ has been demonstrated on six BCP samples, two for each of the $\mathrm{Nb}$ producers (all $\mathrm{Nb}$ sheets have $R R R \approx$ 300). The average value for the BCP samples is $r_{32}=$ $1.86 \pm 0.03$ and hence larger than Ginzburg-Landau value $r_{G L}=1.695$.

If a BCP sample receives an electropolishing of $80 \mu \mathrm{m}$ the ratio $r_{32}$ grows by $\sim 12 \%$. Also a low temperature

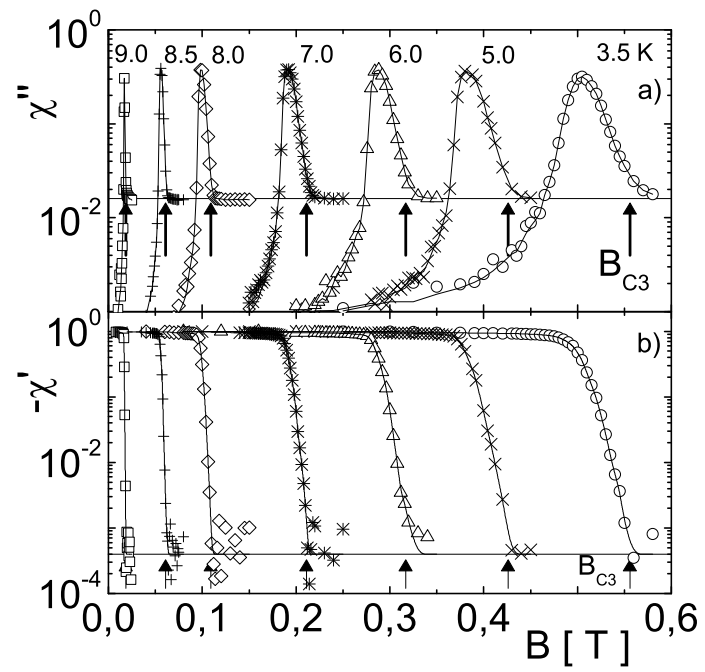

Figure 6: (a) Imaginary and (b) real part of the ac susceptibility of a BCP-treated sample as a function of field for temperatures between $3.5 \mathrm{~K}$ and $9.0 \mathrm{~K}$.

baking (LTB) increases the ratio $r_{32}$ considerably, see Fig. 8. Here the reproducibility has been demonstrated on six BCP samples, two for each of the Nb producers, which were baked for $48 \mathrm{~h}$ at $120^{\circ} \mathrm{C}$ and yielded $r_{32}=$ $2.33 \pm 0.05$. In the bakeout at $123^{\circ} \mathrm{C}$ the ratio $r_{32}$ grows with increasing baking time. At $144^{\circ} \mathrm{C}$ a saturation value of $r_{32}=2.59 \pm 0.05$ is reached after 24 hours without further increase towards longer baking times. The gain in the ratio $r_{32}$ is completely lost if a $1 \mu \mathrm{m}$ surface layer is removed by chemical etching. This proves that only a thin surface layer is modified by the bakeout.

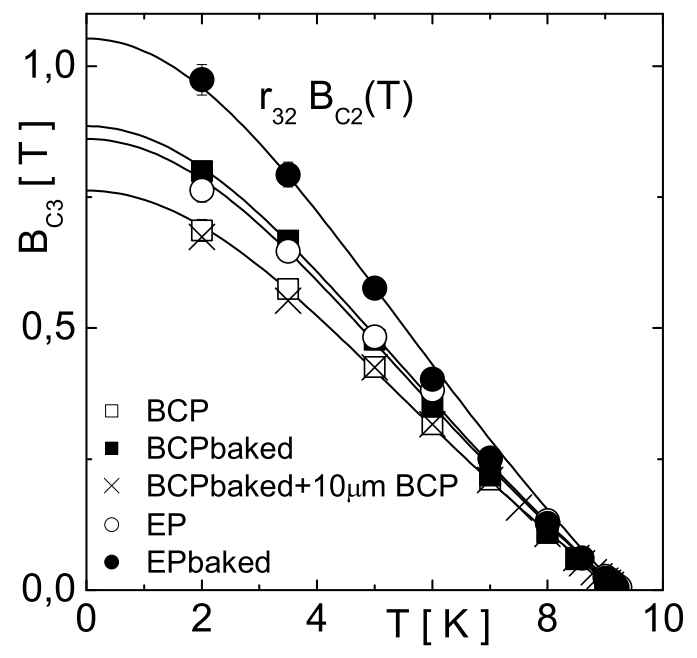

Figure 7: Temperature dependence of critical surface field $B_{c 3}=\mu_{0} H_{c 3}$ of the samples shown in Fig. 5 The data are fitted to the function $B_{c 3}(T)=r_{32} B_{c 2}(T)$, where the ratio $r_{32}$ depends on the surface preparation but not on temperature. This means that $B_{c 2}$ and $B_{c 3}$ have the same temperature dependence. 


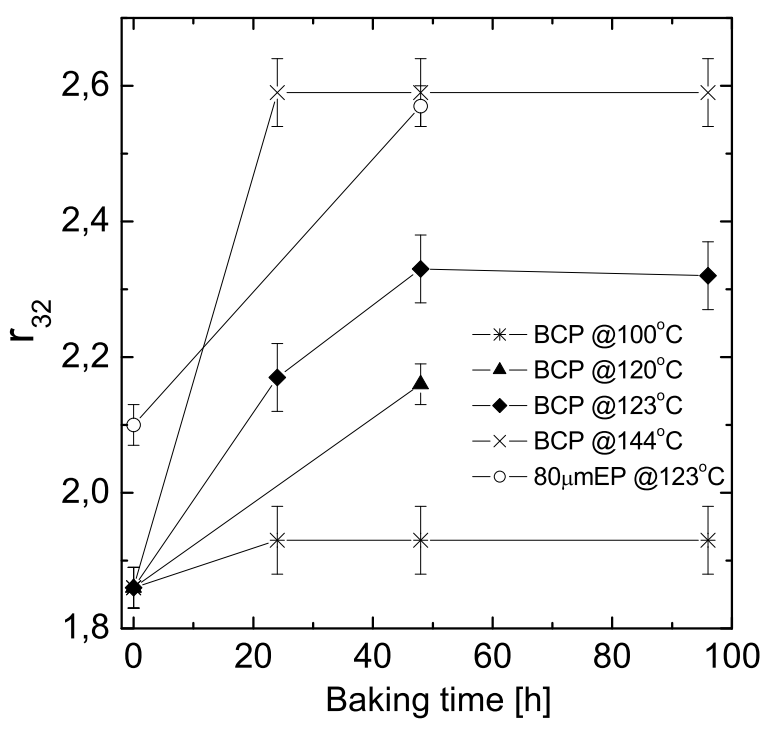

Figure 8: The ratio $r_{32}=B_{c 3} / B_{c 2}$ as a function of baking time for $\mathrm{BCP}$ and $\mathrm{EP}$ samples at different baking temperatures.

Baking at $100^{\circ} \mathrm{C}$ yields only a slight rise in $r_{32}$. Moreover, no appreciable change is observed by extending the baking time from $24 \mathrm{~h}$ to $94 \mathrm{~h}$. This means that $100^{\circ} \mathrm{C}$ is probably too low a temperature for changing the superconductor properties at the Nb surface significantly.

The existence of a critical surface field exceeding the upper critical field of the bulk, $B_{c 3}=r_{G L} B_{c 2}$ with $r_{G L}=1.695$, was predicted by de Gennes and SaintJames by solving the linearized Ginzburg-Landau equation in the presence of a plane boundary which creates a mirror image of the potential. If we retain this idea the increased ratio $r_{32}>r_{G L}$ may be explained by assuming a larger value of $B_{c 2}$ close to the surface, $B_{c 2}^{\text {surf }}>B_{c 2}^{\text {bulk }}$, and by relating $B_{c 3}$ to this field: $B_{c 3}=r_{G L} B_{c 2}^{\text {surf }}$. The enhanced $B_{c 2}^{\text {surf }}$ may be caused for instance by a higher concentration of impurities and a reduced coherence length.

One obvious contaminant is oxygen. After the discovery of the bakeout effect in cavities systematic studies have been conducted on the morphological and chemical structure of niobium samples, for instance by surfacesensitive methods such as XPS (X-ray induced photoelectron spectroscopy) [31-33]. These studies indicate that baking causes a partial disintegration of the external $\mathrm{Nb}_{2} \mathrm{O}_{5}$ layer and the formation of metallic suboxides $\left(\mathrm{NbO}, \mathrm{NbO}_{2}\right)$.

The effect of interstitial oxygen on the upper critical field of niobium was studied by Koch et al. [34]. The concentration $c_{\mathrm{O}}$ of oxygen in atomic per cent can be derived from an expression valid at $4.2 \mathrm{~K}$ [35]

$$
c_{\mathrm{O}}[\text { at. \% }]=1.475 \cdot 10^{-3}\left(B_{c 2}^{\text {surf }}[\mathrm{mT}]-276\right) .
$$

Under the assumption that the enhanced surface fields
Table 3: The ratio $r_{32}=B_{c 3} / B_{c 2}$ for all samples.

\begin{tabular}{|c|c|}
\hline Sample & $r_{32}=B_{c 3} / B_{c 2}$ \\
\hline BCP only & $1.86 \pm 0.03$ \\
\hline \multicolumn{2}{|l|}{$\overline{B C P}+$ Baking } \\
\hline $\mathrm{BCP}+24 \mathrm{~h} 100^{\circ} \mathrm{C}$ & $1.93 \pm 0.05$ \\
\hline $\mathrm{BCP}+48 \mathrm{~h} 100^{\circ} \mathrm{C}$ & $1.93 \pm 0.05$ \\
\hline $\mathrm{BCP}+96 \mathrm{~h} 100^{\circ} \mathrm{C}$ & $1.93 \pm 0.05$ \\
\hline $\mathrm{BCP}+48 \mathrm{~h} 120^{\circ} \mathrm{C}$ & $2.16 \pm 0.03$ \\
\hline $\mathrm{BCP}+24 \mathrm{~h} 123^{\circ} \mathrm{C}$ & $2.17 \pm 0.05$ \\
\hline $\mathrm{BCP}+48 \mathrm{~h} 123^{\circ} \mathrm{C}$ & $2.33 \pm 0.05$ \\
\hline $\mathrm{BCP}+96 \mathrm{~h} 123^{\circ} \mathrm{C}$ & $2.32 \pm 0.05$ \\
\hline $\mathrm{BCP}+24 \mathrm{~h} 144^{\circ} \mathrm{C}$ & $2.59 \pm 0.05$ \\
\hline $\mathrm{BCP}+48 \mathrm{~h} 144^{\circ} \mathrm{C}$ & $2.59 \pm 0.05$ \\
\hline $\mathrm{BCP}+96 \mathrm{~h} 144^{\circ} \mathrm{C}$ & $2.59 \pm 0.05$ \\
\hline \multicolumn{2}{|c|}{$\overline{\mathrm{BCP}+\text { Baking}+\mathrm{BCP}}$} \\
\hline $\mathrm{BCP}+48 \mathrm{~h} 120^{\circ} \mathrm{C}+1 \mu \mathrm{m} \mathrm{BCP}$ & $1.90 \pm 0.03$ \\
\hline$\overline{\mathrm{BCP}}+48 \mathrm{~h} 120^{\circ} \mathrm{C}+5 \mu \mathrm{m} \mathrm{BCP}$ & $1.87 \pm 0.03$ \\
\hline$\overline{\mathrm{BCP}}+48 \mathrm{~h} 120^{\circ} \mathrm{C}+10 \mu \mathrm{m} \mathrm{BCP}$ & $1.86 \pm 0.03$ \\
\hline \multicolumn{2}{|l|}{$\mathrm{BCP}+\mathrm{EP}$} \\
\hline $\mathrm{BCP}+40 \mu m \mathrm{EP}$ & $1.92 \pm 0.05$ \\
\hline $\mathrm{BCP}+80 \mu m \mathrm{EP}$ & $2.10 \pm 0.03$ \\
\hline $\mathrm{BCP}+145 \mu m \mathrm{EP}$ & $1.99 \pm 0.05$ \\
\hline $\mathrm{BCP}+165 \mu \mathrm{mEP}$ & $1.99 \pm 0.05$ \\
\hline \multicolumn{2}{|c|}{$\mathrm{BCP}+\mathrm{EP}+$ Baking } \\
\hline $\mathrm{BCP}+40 \mu m \mathrm{EP}+48 \mathrm{~h} 123^{\circ} \mathrm{C}$ & $2.64 \pm 0.05$ \\
\hline $\mathrm{BCP}+80 \mu m \mathrm{EP}+48 \mathrm{~h} 123^{\circ} \mathrm{C}$ & $2.57 \pm 0.05$ \\
\hline $\mathrm{BCP}+145 \mu m \mathrm{EP}+48 \mathrm{~h} 123^{\circ} \mathrm{C}$ & $2.40 \pm 0.05$ \\
\hline $\mathrm{BCP}+165 \mu m \mathrm{EP}+48 \mathrm{~h} 123^{\circ} \mathrm{C}$ & $2.40 \pm 0.05$ \\
\hline
\end{tabular}

$B_{c 2}^{\text {surf }}$ are indeed caused by oxygen this formula is used to compute the oxygen concentration listed in Table 4 Figure 9 shows that the oxygen concentration increases if a BCP sample is subjected to EP or LTB.

In a study on $\mathrm{BCP}$ cavities recently performed at Jefferson Lab, Ciovati et al. [16] measured an average surface $R R R=206 \pm 3$. Under the same assumption that oxygen is the main contaminant they find $c_{O}=0.017$ at.\% which, considering the different $\mathrm{BCP}$ conditions in the two laboratories, is in reasonable agreement with our results for BCP samples of $c_{O}=0.035$ at. $\%$.

Shmidt [36] has proposed a model in which the impurities are contained in a layer whose thickness is less than the coherence length of the bulk $d \leq \xi$. This model predicts the following relation:

$$
r_{32}=1.67\left(1+\left(1-\chi_{G}\right) \sqrt{1.7} \frac{d}{\xi(T)}\right) .
$$

Here $\chi_{G}$ is the Gor'kov impurity function which relates the GL $\kappa$ parameters in the pure bulk and the point defected surface, $\chi_{G}=\kappa / \kappa_{\text {surf }}$. Since $\left(1-\chi_{G}\right) \leq 1$ and $d / \xi \leq 1$ the maximum ratio is $r_{\max } \leq 3.8$, which is consistent with our results. Eq. (4.2) embodies two unknowns, the mean free path $\ell$ and and the thickness $d$ along with $\xi_{0}=1.35 \xi(0)$ [37], hence we can only consider 


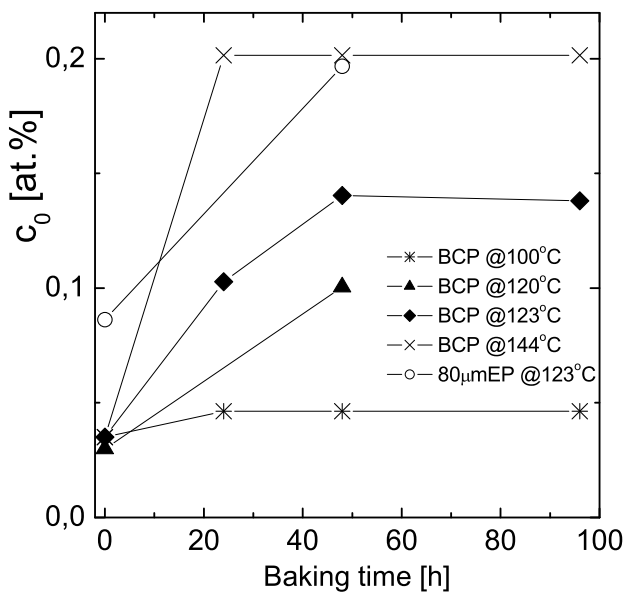

Figure 9: Oxygen concentration as a function of baking time for different surface treatments.

limiting cases. In the dirty limit $\ell \rightarrow 0$ ones gets $\chi_{G} \simeq$ $1.33 \ell / \xi_{0} \rightarrow 0$ and $r_{32}$ increases with increasing thickness $d$ of the contaminated layer. The minimum values of $d$ in the dirty limit are listed in Tab. 4 In the clean limit $\ell \gg \xi_{0}$ one has $\chi_{G} \simeq 1-0.884 \xi_{0} / \ell$, hence the maximum value of $\ell$ is given by putting $d=\xi$. The results are listed in Tab. 四 Both the oxygen contamination model and the Shmidt model are consistent with the idea of oxygen diffusion during baking from the $\sim 5 \mathrm{~nm}$ thin $\mathrm{Nb}_{2} \mathrm{O}_{5}$ sheath into deeper layers [11].

\section{Coherent surface superconduc- tivity}

\subsection{Surface conductivity and resistivity}

We now examine in more detail the behaviour of the niobium samples for applied magnetic dc fields in the range between $B_{c 2}$ and $B_{c 3}$ where only a thin surface sheath is superconducting while the bulk is in the normal state. Coming down from high fields, the screening part of $\chi$ starts to grow upon crossing $B_{c 3}$. Owing to the high sensitivity of the SQUID magnetometer, the onset of screening is clearly visible when $-\chi^{\prime}$ is plotted on a logarithmic scale (Fig. 10). This allows a precise determination of the critical surface field $B_{c 3}$. On a linear scale, however, the onset of surface superconductivity is barely visible, while a strong rise of $-\chi^{\prime}$ is observed at a lower field $B_{c 3}^{c o h}$. Following Ref. [38], we call this field the coherent critical surface field, for reasons explained below. It is still well above the nucleation field $B_{c 2}$ of the bulk.

To gain a deeper insight into the physical mechanisms in this region it is instructive to study the field dependence of the complex conductivity $\sigma=\sigma^{\prime}-i \sigma^{\prime \prime}$ and the resistivity $\rho=1 / \sigma=\rho^{\prime}+i \rho^{\prime \prime}$. These quantities can be calculated
Table 4: Parameters of the oxygen-contamination model and the Shmidt model.

\begin{tabular}{|c|c|c|c|}
\hline Sample & $c_{\mathrm{O}}($ at. $\% \mathrm{O})$ & $d_{\min }[\mathrm{nm}]$ & $\ell_{\max }[\mathrm{nm}]$ \\
\hline BCP only & 0.035 & 2.5 & 436 \\
\hline \multicolumn{4}{|c|}{ BCP+Baking } \\
\hline $24 \mathrm{~h} 100^{\circ} \mathrm{C}$ & 0.052 & 3.5 & 318 \\
\hline $48 \mathrm{~h} 100^{\circ} \mathrm{C}$ & 0.052 & 3.5 & 318 \\
\hline $96 \mathrm{~h} 100^{\circ} \mathrm{C}$ & 0.052 & 3.5 & 318 \\
\hline $48 \mathrm{~h} 120^{\circ} \mathrm{C}$ & 0.106 & 6.5 & 169 \\
\hline $24 \mathrm{~h} 123^{\circ} \mathrm{C}$ & 0.109 & 6.5 & 166 \\
\hline $48 \mathrm{~h} 123^{\circ} \mathrm{C}$ & 0.147 & 8.5 & 125 \\
\hline $96 \mathrm{~h} 123^{\circ} \mathrm{C}$ & 0.144 & 8.5 & 127 \\
\hline $24 \mathrm{~h} 144^{\circ} \mathrm{C}$ & 0.209 & 12 & 90 \\
\hline $48 \mathrm{~h} 144^{\circ} \mathrm{C}$ & 0.209 & 12 & 90 \\
\hline $96 \mathrm{~h} 144^{\circ} \mathrm{C}$ & 0.209 & 12 & 90 \\
\hline \multicolumn{4}{|c|}{$\mathrm{BCP}+$ Baking $\left(48 \mathrm{~h}\right.$ at $\left.120^{\circ} \mathrm{C}\right)+$ short $\mathrm{BCP}$} \\
\hline $1 \mu \mathrm{m} \mathrm{BCP}$ & 0.045 & 3. & 360 \\
\hline $5 \mu \mathrm{m} \mathrm{BCP}$ & 0.037 & 2.5 & 414 \\
\hline $10 \mu \mathrm{m} \mathrm{BCP}$ & 0.035 & 2.5 & 436 \\
\hline \multicolumn{4}{|c|}{$\mathrm{BCP}+\mathrm{EP}$} \\
\hline $40 \mu \mathrm{m} \mathrm{EP}$ & 0.049 & 3 & 331 \\
\hline $80 \mu \mathrm{m} \mathrm{EP}$ & 0.092 & 6 & 192 \\
\hline $145 \mu \mathrm{m} \mathrm{EP}$ & 0.066 & 4 & 259 \\
\hline $165 \mu \mathrm{m} \mathrm{EP}$ & 0.066 & 4 & 259 \\
\hline \multicolumn{4}{|c|}{$\mathrm{BCP}+\mathrm{EP}+$ Baking $\left(48 \mathrm{~h}\right.$ at $\left.123^{\circ} \mathrm{C}\right)$} \\
\hline $40 \mu \mathrm{m} \mathrm{EP}$ & 0.221 & 13 & 85 \\
\hline $80 \mu \mathrm{m} \mathrm{EP}$ & 0.204 & 12 & 92 \\
\hline $145 \mu \mathrm{m} \mathrm{EP}$ & 0.163 & 9.5 & 113 \\
\hline $165 \mu \mathrm{m} \mathrm{EP}$ & 0.163 & 9.5 & 113 \\
\hline
\end{tabular}

from the ac susceptibility with the help of Eq. (3.1). In the range $B_{c 3}>B>B_{c 3}^{c o h}$ the resistivity $\rho^{\prime}$ is sharply dropping with decreasing $B$, but there is only very little screening ( $\sigma^{\prime \prime}$ is extremely small), as can be seen from Fig.11] This can be interpreted in the following way: for applied fields $B_{c 3}>B>B_{c 3}^{c o h}$ the surface sheath is not uniformly superconducting but only in disconnected domains such that super-currents flowing around the entire $\mathrm{Nb}$ cylinder are impeded. The situation changes dramatically when the applied field falls below $B_{c 3}^{c o h}$ : here the Ohmic resistivity $\rho^{\prime}$ vanishes and the imaginary part $\sigma^{\prime \prime}$ of the conductivity rises steeply with decreasing field. Since $\omega \sigma^{\prime \prime}$ is proportional to the Cooper pair density ${ }^{4}$, it was proposed in Ref. [38] to associate $B_{c 3}^{c o h}$ with the onset of long-range superconductivity in the surface sheath. This observation justifies the name coherent surface field. Slightly below $B_{c 3}^{c o h}$ the susceptibility reaches the value $\chi^{\prime}=-1$ which is characteristic of complete shielding.

The singular behaviour of $\rho^{\prime}$ and $\sigma^{\prime \prime}$ near the transition to coherent surface superconductivity can be described by power laws in $\left|B-B_{c 3}^{c o h}\right|$, see Fig. 11] Above the

\footnotetext{
${ }^{4}$ In the two-fluid model of superconductivity, the imaginary part of the ac conductivity is given by $\sigma^{\prime \prime}=2 n_{c} e^{2} /\left(m_{e} \omega\right)$ where $n_{c}$ is the Cooper pair density, see for example [39].
} 


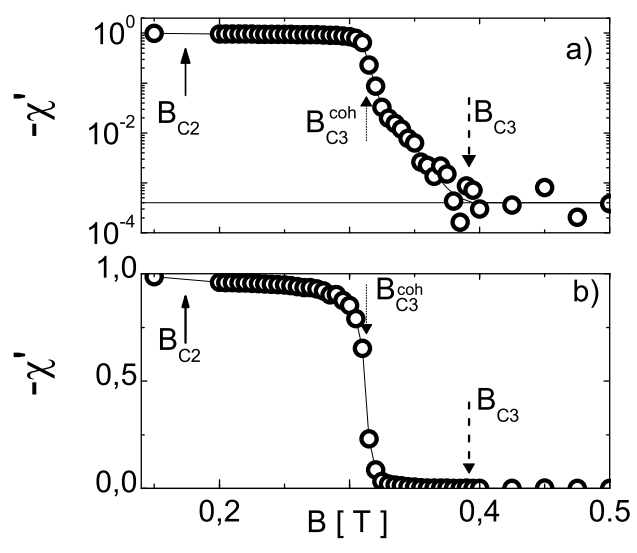

Figure 10: a) The negative real part of the susceptibility of an EP cylinder at $6.0 \mathrm{~K}$ and $10 \mathrm{~Hz}$, plotted on a logarithmic scale, as a function of the applied field. The start of the growth of $\left|\chi^{\prime}\right|$ defines the critical surface field $B_{c 3}=\mu_{0} H_{c 3}$. b) The same data on a linear scale. The field $B_{c 3}^{c o h}$ marks the onset of coherent surface superconductivity with a strong growth of $\left|\chi^{\prime}\right|$ towards $\left|\chi^{\prime}\right|=1$.

transition one gets

$$
\rho^{\prime}(B) \propto\left(B-B_{c 3}^{c o h}\right)^{s} \quad \text { for } \quad B_{c 3}^{c o h}<B<B_{c 3}
$$

while below the transition

$$
\sigma^{\prime \prime}(B) \propto\left(B_{c 3}^{c o h}-B\right)^{t} \quad \text { for } \quad B_{c 2}<B<B_{c 3}^{c o h} .
$$

For the EP samples (baked and unbaked) the exponents are found to be equal: $s=t=1.3 \pm 0.1$, see Figs. 11 and 12 This is consistent with a 2-D model of a percolation-driven transition to coherent superconductivity $[38,40,41]$. For the BCP samples, however, the exponents $s=1.05 \pm 0.10$ and $t=1.4 \pm 0.1$ are different (see Fig. 13), indicating a dimensionality of the percolating network which is slightly higher than two but still much smaller than three [42]. The power law analysis points to different surface topologies and electronic structures of BCP and EP samples. As expected, baking has no influence on the topology, and hence does not modify the exponents.

An interesting observation is that the coherent critical surface field is always a fixed fraction of the GinzburgLandau-type surface field: $B_{c 3}^{c o h}=(0.81 \pm 0.02) B_{c 3}$, independent of the surface topology, see Fig. 14 This suggests some intrinsic but yet unknown effect behind the formation of the coherent surface superconductivity.

\subsection{Frequency dependence of the con- ductivity}

Up to now we have discussed the ac susceptibility and conductivity in the quasi-static limit at $f=10 \mathrm{~Hz}$. The investigations have been extended up to $1 \mathrm{MHz}$. In Fig. 15] the quantity $\omega \sigma^{\prime \prime}$, which is a measure of the superfluid density, and the loss component $\sigma^{\prime}$ are shown

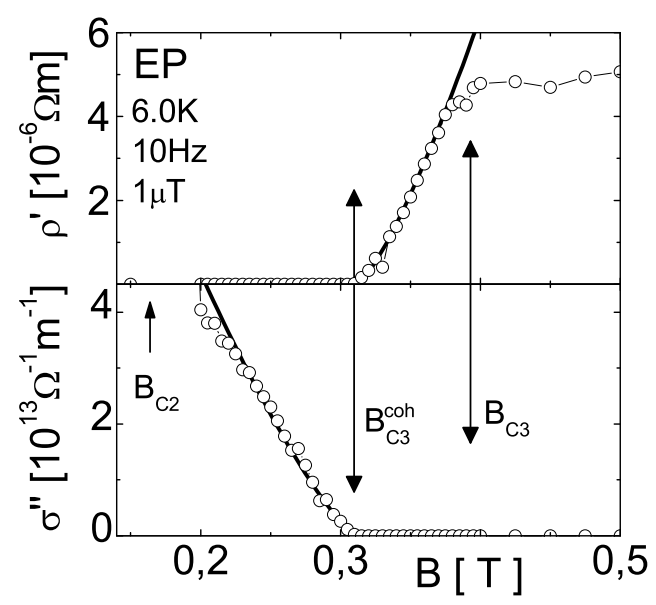

Figure 11: Top: The real part of the resistivity $\left|\rho^{\prime}\right|$ of an EP sample as a function of field. For $B>B_{c 3}^{c o h}$ the data are well described by the power law curve $\rho^{\prime} \propto\left|B-B_{c 3}^{c o h}\right|^{1.3}$. Bottom: The imaginary part of the conductivity in comparison with the power law $\sigma^{\prime \prime} \propto\left|B_{c 3}^{c o h}-B\right|^{1.3}$.

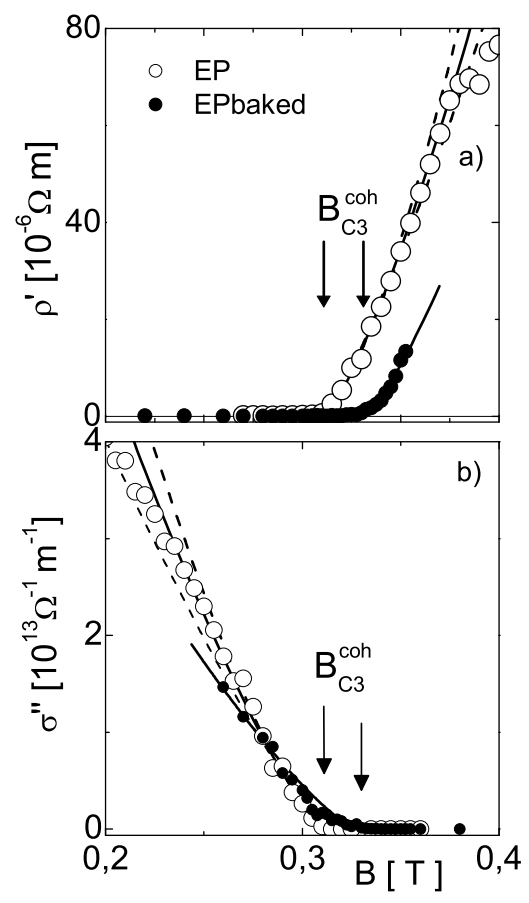

Figure 12: Analysis of the singular behaviour (a) of the real part of the resistivity $\rho^{\prime}$ and (b) of the imaginary part of the conductivity $\sigma^{\prime \prime}$ of an electropolished $\mathrm{Nb}$ cylinder at $6 \mathrm{~K}$. Open circles: before baking, closed circles: after baking. The solid curves are power-law fits revealing the same exponents before and after baking. Note that the coherent surface field is larger after baking. 


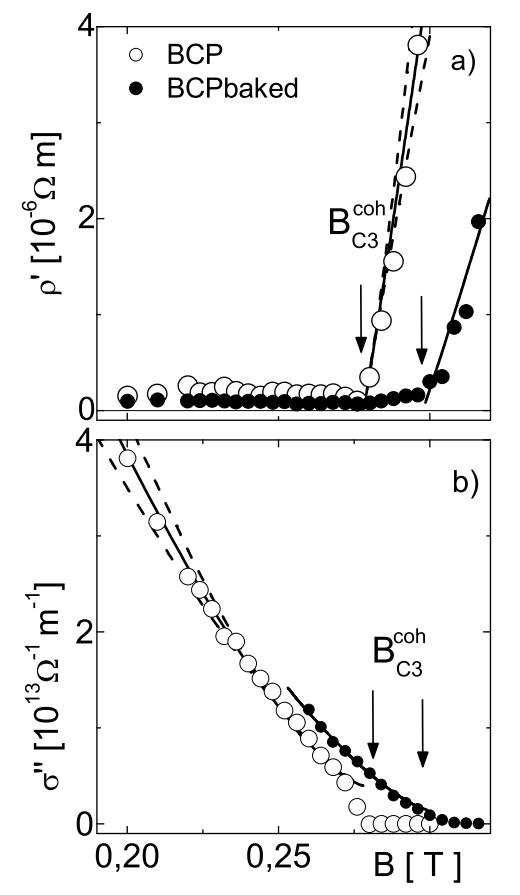

Figure 13: (a) The real part of the resistivity $\rho^{\prime}$ and (b) the imaginary part of the conductivity $\sigma^{\prime \prime}$ of a chemically etched (BCP) cylinder. Open circles: before baking, closed circles: after baking. Test temperature $6 \mathrm{~K}$.



Figure 14: Linear relation between $B_{c 3}^{c o h}$ and $B_{c 3}$ obtained for samples with different surface treatment. The data point labelled "X" refers to a Nb single crystal with an etched surface [40]. as functions of the applied field for frequencies between $10 \mathrm{~Hz}$ and $1 \mathrm{MHz}$. In the normal conducting regime above $B_{c 3}$ we find that $\sigma^{\prime}=\sigma_{n}=(2.1 \pm 0.3) 10^{9}(\Omega \mathrm{m})^{-1}$ is frequency independent and also field independent since it agrees with the value obtained in zero field (see Sect. 2]. Below $B_{c 3}, \sigma^{\prime}$ becomes frequency dependent.

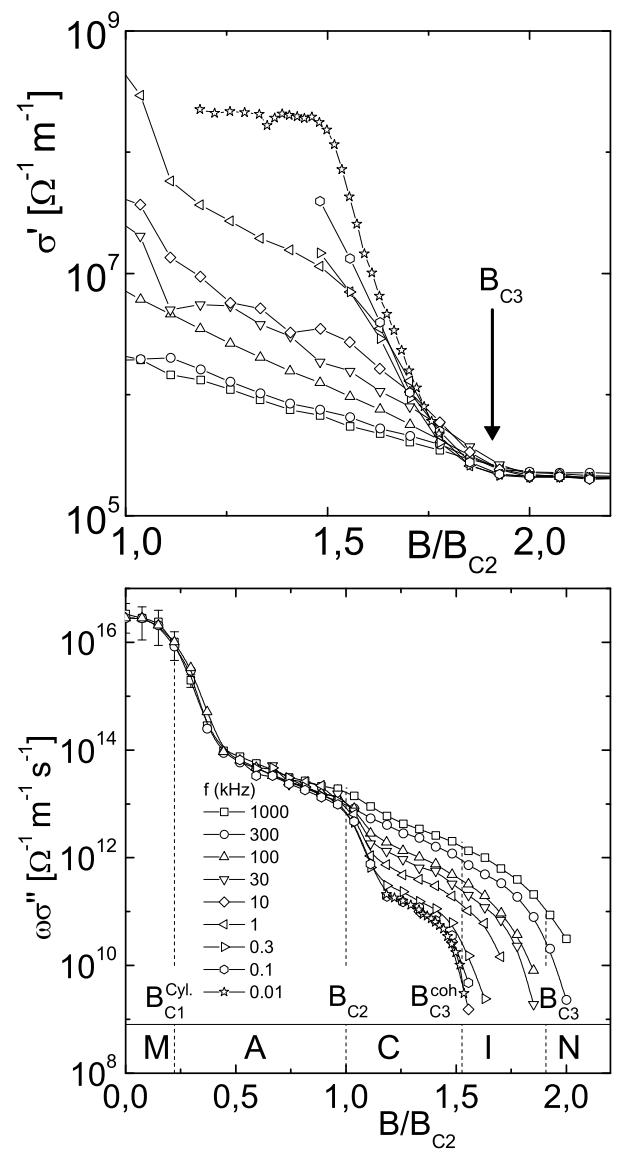

Figure 15: The complex conductivity $\sigma(\omega)$ as a function of field for frequencies between $10 \mathrm{~Hz}$ and $1 \mathrm{MHz}$. The top graph shows $\sigma^{\prime}$, the bottom graph $\omega \sigma^{\prime \prime}$. The indicated critical fields $B_{c 1}, B_{c 2}, B_{c 3}^{c o h}$ and $B_{c 3}$ separate five phases: Meissner M, Abrikosov A, coherent surface superconductivity $\mathrm{C}$, incoherent surface superconductivity I, normal state N.

From the field dependence of $\omega \sigma^{\prime \prime}$ it is possible to distinguish five phases of the samples obtained by sweeping the dc magnetic field [41]. The indicated critical fields separate the Meissner phase $(\mathrm{M})$, the Abrikosov vortex lattice phase $(A)$, the coherent surface phase $(C)$, the incoherent surface phase (I), and finally the normal phase $(\mathrm{N})$. The two surface phases are depicted schematically in Fig. 16] The Meissner and Abrikosov phase are frequency independent. The strongest frequency variation is observed close to the coherent surface field $B_{c 3}^{c o h}$.

\subsection{Surface critical currents}

As a further evidence for the coherence of the surface superconductivity below $B_{c 3}^{c o h}$, a finite critical current has 


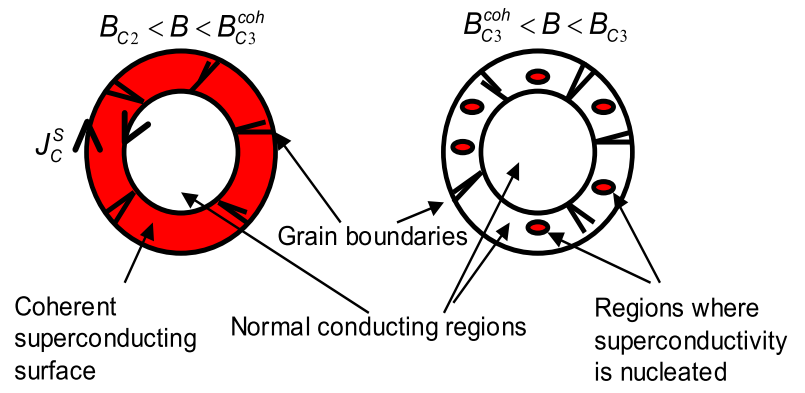

Figure 16: Schematic view of the coherent and incoherent surface phases of a superconducting cylinder.

been detected $[38,40,41]$. To this end, a small longitudinal gradient in the dc magnetic field $B$ of the SQUID magnetometer has been utilized, the field inhomogeneity being $0.0025 \% / \mathrm{mm}$. During the motion of the sample through the pickup coil with a velocity $v_{z}$ the field gradient induces an azimuthal electric field along the circumference of the cylinder of radius $a$

$$
E_{\phi}=\frac{a v_{z}}{2} \cdot \frac{d B}{d z} .
$$

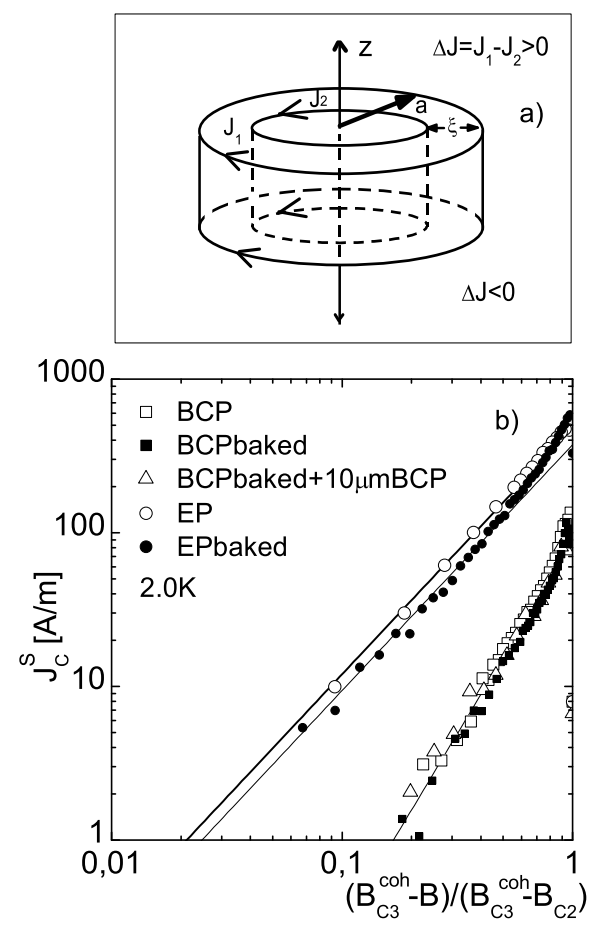

Figure 17: a) Schematics of the surface supercurrents according to Fink and Barnes [44]. b) Dependence of the surface current per unit length $J_{c}^{s}$ on the normalized magnetic field $\left(B_{c 3}^{c o h}-B\right) /\left(B_{c 3}^{c o h}-B_{c 2}\right)$ for all samples. $(T=2 \mathrm{~K})$.

Depending on the direction of motion, the response is either diamagnetic or paramagnetic. These responses are symmetric with respect to the linear background

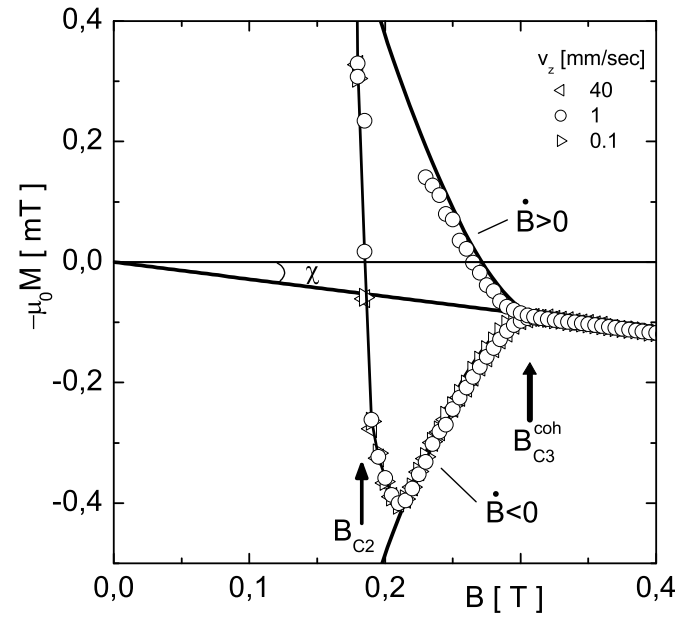

Figure 18: Magnetization of an EP cylinder between $B_{c 2}$ and $B_{c 3}^{c o h}$ at $6.0 \mathrm{~K}$ measured along positive and negative field gradients. Above $B_{c 2}$, the magnetization is independent of the scan velocity $v_{z}$. Note the small values of the magnetization.

$M_{e l}(H)=\chi_{e l} B / \mu_{0}$ due to the paramagnetism of the normal conduction electrons in niobium (see sect. 6). The induced surface current per unit length

$$
J_{c}^{s}(B)=M-\chi_{e l} B / \mu_{0}
$$

is shown in Fig. 17 for all samples as a function of the reduced field $\left(B_{c 3}^{c o h}-B\right) /\left(B_{c 3}^{c o h}-B_{c 2}\right)$ at $2.0 \mathrm{~K}$. An important observation is that $J_{c}^{s}(B)$ does not depend on the scan velocity $v_{z}$, which has been varied between $0.1 \mathrm{~mm} / \mathrm{s}$ and $40 \mathrm{~mm} / \mathrm{s}$ (see Fig. 18). This indicates that $E_{\phi}$ is always large enough to excite the critical surface supercurrent density in the cylinder, $J_{c}^{s}(B)$.

According to Abrikosov [43] the surface current density (current per unit length) should obey a power law

$$
J_{c}^{s}(B) \propto\left(1-\frac{B}{B_{c 3}}\right)^{v}
$$

with $v=1.5$. For the EP samples we find an exponent $v=1.6 \pm 0.1$, consistent with the Abrikosov calculation. The measured currents per unit length of $477 \pm 24 \mathrm{~A} / \mathrm{m}$ (unbaked) and $374 \pm 24 \mathrm{~A} / \mathrm{m}$ (baked) are in good agreement with predictions by Fink and Barnes [44] who consider a multiply connected surface sheath with two currents flowing in the opposite direction as illustrated by Fig. 17h. In this sense, our surface current (5.3) must be interpreted as the difference between two large counterrotating currents. We observe a clear difference between electropolished and chemically etched samples: the BCP cylinders have a factor of six smaller critical surface currents and the exponent is larger, $v=2.5 \pm 0.3$.

Following Ref. [38] we compare the exponent $v$ with the scaling prediction for a multidimensional percolation network, $v=t-\Delta[45]$. For the EP sample the magnitude of the so called twistedness index of the macrobond, $\Delta=t-v=-0.3 \pm 0.3$ is in agreement with the small 
and negative value found for 2-D granular superconducting PbGe films [45]. The higher value of $\Delta$ for the $\mathrm{BCP}$ samples indicates that the surface currents have to follow more complicated orbits than in EP samples. The lower surface current densities $J_{c}^{s}$ in BCP-treated surfaces and the higher twistedness index $\Delta$ are possibly related to the larger roughness at the grain boundaries.

The surface currents per unit length, as measured in our experiment, are three orders of magnitude lower than the currents that would be needed to excite a niobium cavity to $\mathrm{rf}$ magnetic fields beyond $H_{c 2} \approx 3 \cdot 10^{5} \mathrm{~A} / \mathrm{m}$. However, our quasi-static measurements $(f=10 \mathrm{~Hz})$ do not exclude the possibility that much higher surface currents might exist in the non-equilibium state of superconductivity which is relevant for high-frequency cavities.

\section{Paramagnetic susceptibility}

By measuring the magnetic susceptibility in a large external field $B=0.7 \mathrm{~T}$ where the entire sample is in the normal state it is possible to search for magnetic impurities in the niobium. The data are shown in Fig. 19] for temperatures between 2 and $300 \mathrm{~K}$. Above $50 \mathrm{~K}$ our results on the paramagnetic susceptibility $\chi_{e l}$ of the normal conduction electrons in $\mathrm{Nb}$ are in excellent agreement with previous measurements by Hechtfischer [46]. Below $50 \mathrm{~K}$ an additional contribution to $\chi$ is observed, which obeys a Curie-Weiss law

$$
\chi-\chi_{e l}=C /(T-\theta),
$$

see Fig. 19b. The fit values for the Curie constant $C$ and the Curie-Weiss temperature $\theta$ are listed in Tab. Such behaviour indicates the presence of localized magnetic impurities. While $\theta \simeq-1 \mathrm{~K}$ for all samples, the Curie constant, being proportional to the concentration of the localized magnetic moments, increases during the bakeout.

Table 5: Curie constant $C$ and Curie-Weiss temperature $\theta$.

\begin{tabular}{|l|c|c|}
\hline Sample & $C[\mu \mathrm{K}]$ & $\theta[\mathrm{K}]$ \\
\hline BCP & $72.3 \pm 0.1$ & $-0.5 \pm 0.2$ \\
\hline BCP baked & $100.6 \pm 0.07$ & $-2.2 \pm 0.1$ \\
\hline EP & $40.2 \pm 0.3$ & $-0.8 \pm 0.2$ \\
\hline EP baked & $71.0 \pm 0.1$ & $-1.5 \pm 0.2$ \\
\hline BCP baked $+10 \mu m$ BCP & $48.3 \pm 0.4$ & $-1.7 \pm 0.3$ \\
\hline
\end{tabular}

Oxygen vacancies in the $\mathrm{Nb}_{2} \mathrm{O}_{5}$ sheath would be a good candidate for localized magnetic moments [47]. Their Curie-Weiss behaviour has been observed in $\mathrm{Nb}_{2} \mathrm{O}_{5-\delta}$ crystallographic shear structures with $C \simeq 10 \mathrm{mK}$ for $\delta \approx 0.17$ [47]. Assuming the shear structure to be present in the $d \simeq 5 \mathrm{~nm}$ thin $\mathrm{Nb}_{2} \mathrm{O}_{5}$ layer, one expects a Curie constant $C=3 d / a \cdot 10 \mathrm{mK} \approx 0.12 \mu \mathrm{K}$. Since this is more than two orders of magnitude smaller than the values in


Figure 19: a) Temperature dependence of the susceptibility of all samples measured at $B=700 \mathrm{mT}$. b) Curie-Weiss contribution to the paramagnetic susceptibility. Solid lines are fits to the Curie-Weiss law $\left(\chi-\chi_{e l}\right)^{-1}=(T-\theta) / C$.

Tab. 5 oxygen vacancies in the $\mathrm{Nb}_{2} \mathrm{O}_{5}$ sheath cannot account for the observed magnetic behaviour.

The paramagnetism of other impurities, which may be introduced by the $\mathrm{BCP}$ and $\mathrm{EP}$ processes, like N, C, $\mathrm{F}, \mathrm{P}, \mathrm{S}$, the hydrogen bonded $\mathrm{H}_{2} \mathrm{O} / \mathrm{C}_{x} \mathrm{H}_{y}(\mathrm{OH})_{z}[48]$ and some niobium suboxides $\mathrm{NbO}_{x}(x \lesssim 1)$, has not yet been investigated. Perhaps they form clusters with large paramagnetic moments. After baking, the Curie constant $C$ is found to be increased by about $40-50 \%$. One possibility would be that during baking additional magnetic moments are released from external or internal surfaces.

The observation that a BCP-treated sample has a significantly larger Curie constant than an EP sample indicates that the magnetic moments are not confined to the surface only but reside also deeper in the material, probably in the grain boundaries which are more pronounced in BCP than in EP surfaces. 


\section{Summary and conclusions}

As expected, the bulk properties of the niobium samples, $T_{c}, B_{c}, R R R$ and $B_{c 2}$, remain invariant when different surface treatments such as chemical etching and electropolishing or a low-temperature bakeout are applied. In contrast to this, the superconducting properties of the surface itself are found to be strongly modified by these treatments. Evidence for surface superconductivity at fields exceeding the upper critical field $B_{c 2}$ of the bulk is found in all samples. The critical surface field $B_{c 3}$ is always larger than the value $B_{c 3}=1.695 B_{c 2}$ derived from the Ginzburg-Landau theory, the ratio $r_{32}=B_{c 3} / B_{c 3}$ amounts to 1.86 for BCP samples and 2.1 for EP samples. It increases further by baking the sample at $120-140^{\circ} \mathrm{C}$ for 24 to 96 hours. We interpret this enhanced surface field as being due to increased impurity contents of the niobium in a layer close to the surface and, related to this, a reduced electron mean free path. The most likely contaminant is oxygen.

A most remarkable observation is that two different phases of surface superconductivity exist which are separated by a "coherent" critical surface field $B_{c 3}^{c o h}$ : a coherent phase $\mathrm{C}$ for applied fields between $B_{c 2}$ and $B_{c 3}^{c o h}$ with bipolar shielding currents going around the whole cylindrical sample, and an incoherent phase I between $B_{c 3}^{c o h}$ and $B_{c 3}$ which is characterized by disconnected superconducting regions with normal zones in between. Both $B_{c 3}^{c o h}$ and $B_{c 3}$ depend on the surface preparation but the ratio $B_{c 3}^{c o h} / B_{c 3}$ has the value 0.81 for all samples: $\mathrm{BCP}$, $\mathrm{EP}$, unbaked and baked. A power-law analysis of the complex conductivity and resistivity reveals that at $B_{c 3}^{c o h}$ a phase transition takes place between coherent and incoherent surface superconductivity. For the EP samples the exponents are in agreement with the expectation for percolation through a two-dimensional network of superconducting and resistive sections. A different behaviour is seen in the BCP samples, here the dimensionality of the network would have to be slightly larger that two. We suspect that this may be related to weak links at the grain boundaries and to more complicated, nonplanar current paths in the surface layer.

In the coherent phase $\mathrm{C}$, a small net current around the $\mathrm{Nb}$ cylinder can be induced by a time-varying magnetic field whose direction depends on the sign of $\dot{B}$ according to Lenz's rule. However, this net current, being the difference of two counter-rotating currents, is only a few 100 $\mathrm{A} / \mathrm{m}$ and thereby three orders lower than the rf currents that would be needed to operate an accelerating cavity at rf magnetic fields above $H_{c 2} \approx 3 \cdot 10^{5} \mathrm{~A} / \mathrm{m}$. Our steady-state results do not exclude the possibility that higher surface currents might exist in non-equilibrium states, for instance in the high-frequency fields applied to accelerator cavities. In the BCP samples the surface currents are a factor of six lower which again points to weak links at grain boundaries.

An important result of our investigations is that the various surface preparation steps improving cavity perfor- mance have all a well-measurable influence on the magnetic properties of the samples. Electrolytic polishing of a BCP sample raises the critical surface field $B_{c 3}$ by about $10 \%$. The low-temperature baking leads to a further enhancement by $20 \%$. The critical exponents of the power law fits near $B_{c 3}^{c o h}$ are different for EP- and BCP-treated samples: we get $s=t=1.3 \pm 0.1$ for EP and $s=1.05 \pm 0.1, t=1.4 \pm 0.1$ for BCP samples. From this one can conclude that the smooth EP surface is able to support planar (two-dimensional) surface currents while the rough grain boundaries in a BCP surface enforce more complicated current patterns. The EP samples feature a coherent surface phase which resembles the Meissner phase in the bulk. In the BCP samples this coherent phase is disturbed by weak links at the grain boundaries.

The paramagnetic susceptibility in the normalconducting regime is dominated by the normal conduction electrons in niobium but at low temperatures an additional contribution is observed which obeys a Curie-Weiss law and can be attributed to paramagnetic impurities. Their number is increased by low-temperature baking (LTB). This in qualitative accordance with the generally accepted interpretation that LTB leads to a partial reduction of the $\mathrm{Nb}_{2} \mathrm{O}_{5}$ layer and an oxygen diffusion into deeper layers. Remarkably, the density of paramagnetic impurities is larger in $\mathrm{BCP}$ than in EP samples, which may be an indication that the impurities in grain boundaries play an important role.

In our view, the above observations are of high relevance for rf cavities and underline the superiority of electropolished surfaces. It must be emphasized, however, that measurements on niobium samples do not render cavity tests superfluous. One essential difference is that the samples are investigated in a large dc background magnetic field with a superimposed small ac field while in the cavities the rf magnetic field assumes large amplitudes. It is by no means obvious that the superconductor responds in the same way to these different conditions. In particular, the dramatic improvement in the high-field performance of EP cavities by applying the low-temperature bakeout could certainly not have been predicted from the $20 \%$ growth of $B_{c 3}$ observed in EPtreated $\mathrm{Nb}$ samples. According to our understanding the underlying mechanisms of the bakeout effect are not yet fully understood.

\section{Acknowledgements}

Thanks are due to R. Anton (University of Hamburg) for his advice and the possibility to use his equipment for sample preparation, to N. Steinhau-Kühl (DESY) for carrying out the $\mathrm{BCP}$ and $\mathrm{EP}$ treatments of the samples, to D. Görlitz (University of Hamburg) for his help and to W. Gil (University of Hamburg) for SEM and AFM measurements on the samples. The work of S.C. was sup- 
ported by the Deutsche Forschungsgemeinschaft through Grant No. CA 284/1-1.

\section{Appendix}

The SQUID-Magnetometer $\mathrm{MPMS}_{2}$ made by Quantum Design is an excellent apparatus to measure the dc magnetisation and the ac susceptibility of samples with a very high sensitivity and over a wide range of magnetic fields and temperatures. A superconducting solenoid produces a dc field up to $\pm 1 \mathrm{~T}$, and a copper coil with 8 turns produces ac-fields from $10 \mathrm{nT}$ up to $0.5 \mathrm{mT}$, with frequencies from $0.01 \mathrm{~Hz}$ up to $1 \mathrm{kHz}$. The temperature can be varied from $2 \mathrm{~K}$ up to $350 \mathrm{~K}$. The measurable range of magnetic moments extends up to $\pm 0.3 \mathrm{Am}^{2}$ with a sensitivity of $10^{-10} \mathrm{Am}^{2}$.

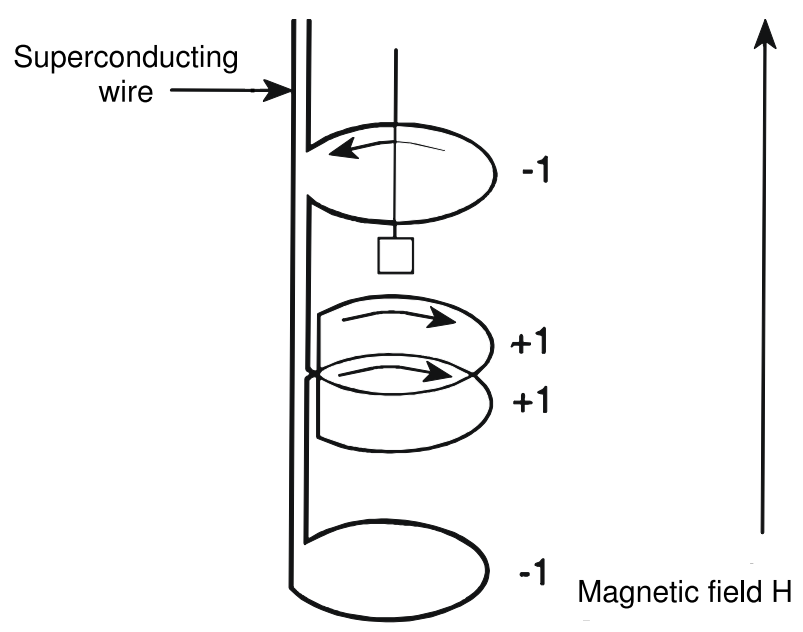

Figure 20: Schematic coil configuration of the SQUID magnetometer.

The detection coil system is built as a gradiometer of second order and made from a single length of superconducting wire. The center coil with two turns is wound clockwise, the upper and lower coils are $14 \mathrm{~mm}$ away from the center coil and consists of a single turn each wound counter-clockwise (see Fig. 20). This configuration cancels noise due to fluctuations in the large magnetic field of the superconducting magnet. Together with a transformer which couples the current changes to the rf-SQUID the pick-up-coil forms a closed superconducting loop.

For dc magnetisation measurements, the sample is moved through the pickup coil at constant speed. The scan length is typically $40 \mathrm{~mm}$, measurements are taken at 30-50 positions. At each position the motion is shortly stopped for the measurement. The current induced in the pickup coil by the moving sample is proportional to the magnetic moment of the sample.

The ac susceptibility is measured at two different positions: in the center coil of the pickup system and in the lower compensation coil. Before applying the ac field a nulling procedure is carried out to remove the induced signal from switching on the superconducting coil.

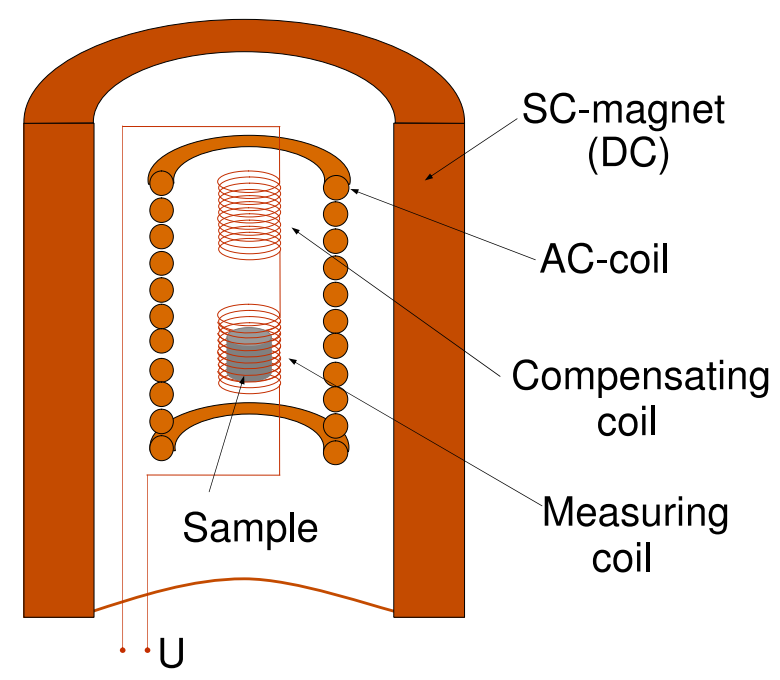

Figure 21: Coil configuration of the mutual inductance magnetometer.

In the second magnetometer, sketched in Fig. 21] the measuring coil system consists of two identical coils wound in opposite direction. Without sample the induced voltage is almost balanced to zero. The sample is positioned in one of the two pickup coils so that the measured voltage is given by the following relation:

$$
U=i I_{a c} \omega M q_{f} \chi
$$

where $I_{a c}=I_{0} \exp (i \omega t)$ is the current in the ac coil, $M$ the mutual inductance coefficient between the ac coil and one of the pickup coils, $q_{f}$ the filling factor given by the ratio of sample volume to coil volume and $\chi$ the ac susceptibility of the sample. Since the two pickup coils are not exactly identical there will be a background voltage $U_{b}$, that depends on the frequency and amplitude of the applied ac field.

$$
\begin{aligned}
U^{\prime} & =\cos \phi\left(U_{m}^{\prime}-U_{b}^{\prime}\right)+\sin \phi\left(U_{m}^{\prime \prime}-U_{b}^{\prime \prime}\right) \\
U^{\prime \prime} & =-\sin \phi\left(U_{m}^{\prime}-U_{b}^{\prime}\right)+\cos \phi\left(U_{m}^{\prime \prime}-U_{b}^{\prime \prime}\right),
\end{aligned}
$$

where $U_{m}^{\prime}$ and $U_{m}^{\prime \prime}$ are the real and imaginary part of the measured signal. The system is calibrated with a spherical $\mathrm{GdCl}_{3} \cdot 6 \mathrm{H}_{2} \mathrm{O}$ sample of $3 \mathrm{~mm}$ diameter. For each ac amplitude and frequency the phase $\phi$ and the mutual coupling $M$ are determined from the conditions $\chi^{\prime \prime}=0$ and $\chi^{\prime}=C / T$, where $C=0.65 \pm 0.01 \mathrm{~K}$ is the known Curie constant of $\mathrm{GdCl}_{3} \cdot 6 \mathrm{H}_{2} \mathrm{O}$.

\section{References}

[1] Gmelin, Handbuch der anorganischen Chemie, Vol. 49(Nb), Springer Verlag Berlin, 1970. 
[2] P. Kneisel, Surface preparations of niobium, in: M. Kuntze (Ed.), Proceedings of the Workshop on RF Superconductivity, Vol. I+II, KFK, Karlsruhe, 1980, p. 27, KFK-3019.

[3] B. Aune, et al., The Superconducting TESLA Cavities, Phys. Rev. ST-AB 3 (9), 092001.

[4] K. Saito, et al., Superiority of Electropolishing over Chemical Polishing on High Gradients, in: Palmieri [14], pp. $759-813$.

[5] K. Saito, et al., High Gradient Performance by Electropolishing with $1300 \mathrm{MHz}$ Single and Multicell Niobium Superconducting Cavities, in: Krawcyk [15], pp. 288-291.

[6] E. Kako, K. Saito, et al., Improvement of Cavity Performance in the Saclay/Cornell/Desy's sc Cavities, in: Krawcyk [15], pp. 179-186.

[7] L. Lilje, et al., Electropolishing and in-situ Baking of $1.3 \mathrm{GHz}$ Niobium Cavities, in: Krawcyk [15], pp. 74-76.

[8] L. Lilje, et al., Improved Surface Treatment of the Superconducting TESLA Cavities, Nucl. Inst. Meth. A $516(2-3)$ (2004) 213-227.

[9] L. Lilje, P. Schmüser, et al.; Achievement of 35 $M V / m$ in the Superconducting Nine-Cell Cavities for TESLA; 2004; accepted for publication in NIM A

[10] J. Knobloch, et al., High field Q slope in SC cavities due to magnetic field enhancement at grain boundaries, in: Krawcyk [15], pp. 77-91.

[11] P. Kneisel, Preliminary experience with "in-situ" baking of niobium cavities, in: Krawcyk [15], pp. 328-335.

[12] P. Kneisel, Surface characterization: What has been done, what has been learned, in: Proceedings of 11th Workshop Superconducting RF, 2003.

[13] B. Visentin, Q-slope at high gradients: Review about experiments and explanations, in: Proceedings of 11th Workshop Superconducting RF, 2003.

[14] V. Palmieri (Ed.), Proceedings of the 8th Workshop on RF Superconductivity, Vol. I+II, INFN, Abano terme, 1997.

[15] F. Krawcyk (Ed.), Proceedings of the 9th Workshop on RF Superconductivity, Vol. I+II, LANL, Santa Fe, 1999.

[16] G. Ciovati, P. Kneisel and G. Myneni, Effect of low temperature baking on niobium cavities, SRF'03, Lübeck/Travemünde, September 2003.
[17] J. Halbritter, Rf residual losses, high electric and magnetic rf fields in superconducting cavities, in Superconducting materials for high energy colliders, edited by L. Cifarelli and L. Maritato, The science and culture series - Physics, pp. 59-79, World Scientific, 1999.

[18] We thank W. Gil (Hamburg) for these data.

[19] L. Lilje, Experimental investigations on superconducting niobium cavities at highest radiofrequency fields, DESY-PhDTHESIS-2001-034.

[20] E. H. Brandt, Superconductors in realistic geometries: geometric edge barrier versus pinning, Physica C 33299 (2000).

[21] D. K. Finnemore, T. F. Stromberg, and C. A. Swenson, Superconducting properties of high-purity niobium, Phys. Rev. 149231 (1966).

[22] E. Maxwell and M. Strongin,Filamentary Structure in Superconductors, Phys. Rev. Lett. 10, 212 (1963)

[23] J. R. Clem, H. R. Kerchner, and S. T. Sekula, ac permeability of defect-free type-II superconductors, Phys. Rev. 141893 (1976).

[24] J. Kötzler et al., Anisotropic dynamical scaling near the vortex-glass transition of twinned $\mathrm{YBa}_{2} \mathrm{Cu}_{3} \mathrm{O}_{7-\delta}$, Phys. Rev. Lett. 72, 2081 (1994).

[25] E. H. Brandt, Superconductor disks and cylinders in an axial magnetic field: II. Non linear and linear ac susceptibilities, Phys. Rev. B 586523 (1998).

[26] M. Tinkham, Effect of Fluxoid Quantization on Transitions of Superconducting Films, Phys. Rev. 129 (1963) 2413.

[27] W. De Sorbo, Effect of Dissolved Gases on Some Superconducting Properties of Niobium, Phys. Rev. 134 (1964) A1119.

[28] D. Saint-James and P. G. de Gennes, Onset of superconductivity in decreasing fields, Phys. Lett. 7 306 (1963).

[29] J. R. Hopkins and D. K. Finnemore, Surface superconductivity in niobium and niobium-tantalum alloys, Phys. Rev. B 9, 108 (1974).

[30] R. W. Rollins and J. Silcox, Nature of the ac transition in the superconducting surface sheath in Pb-2\% In, Phys. Rev. 155404 (1967).

[31] A. Dacca, G. Gemme, L. Mattera, and R. Parodi, $X P S$ analysis of the surface composition of niobium for superconducting RF cavities, Appl. Surf. Sci., 126:219, 1998. 
[32] C. Z. Antoine et al., Alternative Approaches for Surface treatment of $\mathrm{Nb}$ superconducting cavities, In Proceedings of the 9th Workshop on RF Superconductivity [15].

[33] K. Kowalski, XPS measurements at AGH on EP and BCP samples during 'In-Situ' bakeout, Private communication, 2001.

[34] C. Koch, J. O. Scarbrough, and D. Kroeger, Effects of interstitial oxygen on the superconductivity of niobium, Phys. Rev. B, 9:888, 1974.

[35] A. Das Gupta et al., Inhomogeneities in superconducting niobium surfaces, Journal of Appl. Phys. 47, 2146 (1976).

[36] V. V. Shmidt, in Physical metallurgy, physical chemistry and metals physics of superconductors, $\mathrm{p}$. 90 (Moscow: Nauka, in Russian 1967).

[37] L. P. Gor'kov, Theory of superconducting alloys in a strong magnetic field near the critical temperature, JETP 37998 (1960).

[38] J. Kötzler, L. von Sawilski and S. Casalbuoni, Evidence for a percolation-driven transition to coherent surface superconductivity, Phys. Rev. Lett. 92 067005 (2004).

[39] P. Schmüser, Superconductivity in high energy particle accelerators, Prog. Nucl. Part. Phys. 49 (2002) issue 1 and www.desy.de/ pschmues, file supercon_acc.pdf

[40] L. von Sawilski, S. Casalbuoni and J. Kötzler, Surface Superconductivity of Niobium: Onset on longrange coherence, SRF'03, Lübeck/Travemünde, September 2003.

[41] S. Casalbuoni, L. von Sawilski and J. Kötzler, Superconductivity above $H_{c 2}$ as a probe for Niobium RF-cavity surfaces, SRF'03, Lübeck/Travemünde, September 2003.

[42] J. P. Straley, Critical exponents for the conductivity of random resistor lattices, Phys. Rev. B 155733 (1977).

[43] A. A. Abrikosov, Concerning surface superconductivity in strong magnetic fields, JETP 20480 (1965).

[44] H. J. Fink and L. J. Barnes, Critical State of The Superconducting Surface Sheath, Phys. Rev. Lett. 15792 (1965).

[45] G. Deutscher and M. L. Rappaport, Critical currents of superconducting alluminium-germanium and lead-germanium thin film alloys near the metalinsulator transition, J. Phys. Lett. (Paris) 40 L 219 (1979).
[46] D. Hechtfischer, Temperaturabhängigkeit von Knight-shift und Suszeptibilität von Vanadium und Niob, Z. Physik B 23255 (1976).

[47] R. J. Cava et al., Antiferromagnetism and metallic conductivity in $\mathrm{Nb}_{12} \mathrm{O}_{29}$, Nature 350598 (1991); Electrical and magnetic properties of $\mathrm{Nb}_{2} \mathrm{O}_{5-\delta}$ crystallographic shear structures, Phys. Rev. B 446973 (1991).

[48] J.Halbritter, private communication. 